\title{
A CMOS RF tunable filter for TV white space transmitter applications
}

\author{
Jongsik Kim ${ }^{\text {a) }}$ and Hyunchol Shin \\ High-Speed Integrated Circuits and Systems Lab., Kwangwoon University \\ 20 Kwangwoon-Ro, Nowon-gu, Seoul 139-701, Korea \\ a)millitia@kw.ac.kr
}

\begin{abstract}
A UHF-band RF tunable filter is developed for TV white space CMOS transmitter applications. It is based on the combined Sallen-Key and LC notch structure. The inherent transmission zero of the Sallen-Key core is carefully chosen to suppress the $7^{\text {th }}$-harmonic component of the transmitter output, and the LC notch filter is set to suppress the $9^{\text {th }}$-harmonic component. The filter is built in $0.18-\mu \mathrm{m}$ CMOS process and consumes $6.2-\mathrm{mA}$ from a $1.8-\mathrm{V}$ supply. The filter's cutoff frequency is tuned from $100-\mathrm{MHz}$ to $500-\mathrm{MHz}$ in five steps. It is successfully adopted in a TV white space CMOS transmitter integrated circuit.
\end{abstract}

Keywords: RF tunable filter, Sallen-Key filter, harmonic rejection filter

Classification: Integrated circuits

\section{References}

[1] "Second report and order and memorandum opinion and order: Unlicensed operation in the TV broadcast bands," FCC, Washington, DC, FCC ET Docket 04-186, Nov. 2008.

[2] D. McCloskey and P. Gossett, "Wideband Transceiver Architecture for TV Whitespace Applications," Proc. IEEE Symp. New Frontiers in Dynamic Spectrum, pp. 1-7, April 2010.

[3] J. Kim, S. J. Lee, S. Kim, J. O. Ha, Y. S. Eo, and H. Shin, "A 54-862MHz CMOS Transceiver for TV-Band White-Space Device Applications," IEEE Trans. Microw. Theory Tech., to appear in April 2011.

[4] R. F. Wiser, M. Zargari, D. K. Su, and B. A. Wooley, "A 5-GHz wireless LAN transmitter with integrated tunable high- RF filter," IEEE J. SolidState Circuits, vol. 44, no. 8, pp. 2114-2125, Aug. 2009.

[5] P.-U. Su and J.-M. Hsu, "A dual-band enhanced harmonic rejection filter for modulators in GSM and DCS transmitters," Proc. European SolidState Circuits Conf., pp. 663-666, Sept. 2003.

[6] J. A. Weldon, R. S. Nrayanaswami, J. C. Rudell, L. Lin, M. Otsuka, S. Dedieu, L. Tee, K.-C. Tsai, C.-W. Lee, and P. R. Gray, "A $1.75 \mathrm{GHz}$ highly integrated narrowband CMOS transmitter with harmonic rejection mixers," IEEE J. Solid-State Circuits, vol. 36, no. 12, pp. 2003-2015, Dec. 2001. 


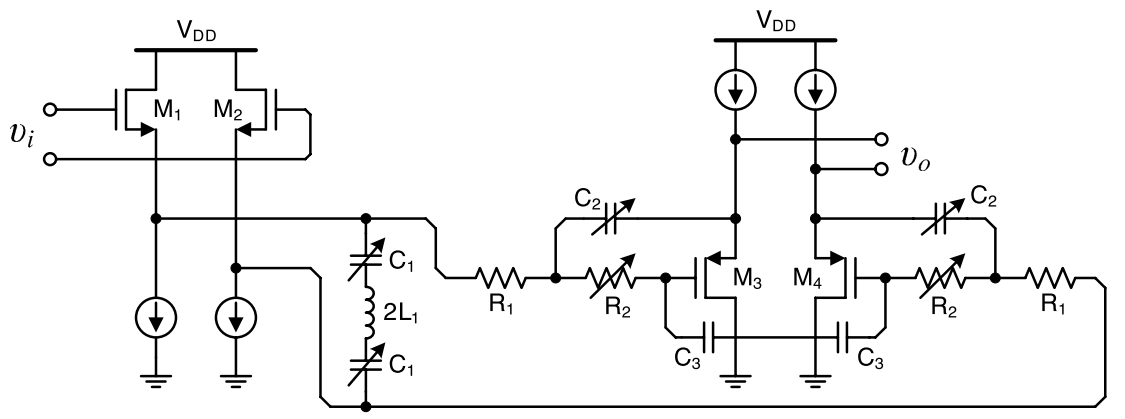

Fig. 1. Circuit schematic of RF tunable filter.

\section{Introduction}

The federal communications commission (FCC) has recently approved the unlicensed radio transmission in the TV white space band for digital data communication. With this, a new path to white space or Super Wi-Fi devices is cleared. The white space device can enable long-distance and robust wireless internet connection since the TV band is better for reaching farther with less attenuation compared to the existing 2.4 or $5 \mathrm{GHz}$ airwaves. Along with IEEE 802.22 cognitive radio based wireless regional area network (WRAN) standardization, the TV-band CMOS transceiver implementation efforts have started to appear in the literature [2, 3].

In order not to interfere with the other TV band signals, the TV white space transmitter requires very low spurious emission level within the band. It can be achieved by embedding an RF tunable filter in the transmitter to reject the harmonic tones that are generated during frequency up-conversion procedure. A $\mathrm{g}_{\mathrm{m}} \mathrm{C}$ filter can be a possible choice for this, but it is very vulnerable to nonlinearity under the transmitter's large voltage swing. A Q-enhanced active LC filter showed excellent selectivity performance, but suffered from narrow band coverage [4]. Another previous approach was to cascade two Sallen-Key stages in series [5]. Although the harmonics were successfully suppressed, its high current consumption due to the two cascaded stages was disadvantageous.

In this work, we present an efficient design approach for the RF tunable filter by combining the Sallen-Key core and an LC notch filter buffered by a source follower. By working with harmonic rejection mixer (HRM) in the transmitter, the RF tunable filter significantly rejects the in-band harmonic emission level in the TV white space transmitter [3].

\section{Circuit design and results}

The RF tunable filter is based on the combined Sallen-Key and LC notch structure. Since a conventional harmonic rejection mixer (HRM) [6] can suppress the $3^{\text {rd }}, 5^{\text {th }}, 11^{\text {th }}$, and $13^{\text {th }}$ harmonics, this $\mathrm{RF}$ tracking filter is designed to reject the $7^{\text {th }}$ and $9^{\text {th }}$ harmonics. The inherent transmission zero of the Sallen-Key structure is beneficial for steep skirt characteristic at around $7^{\text {th }}$ 


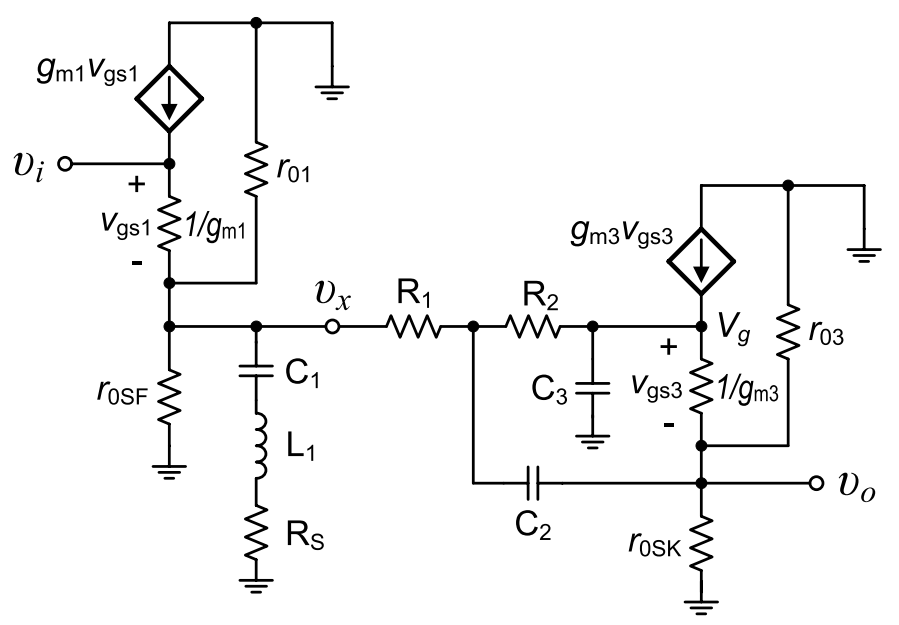

(a)

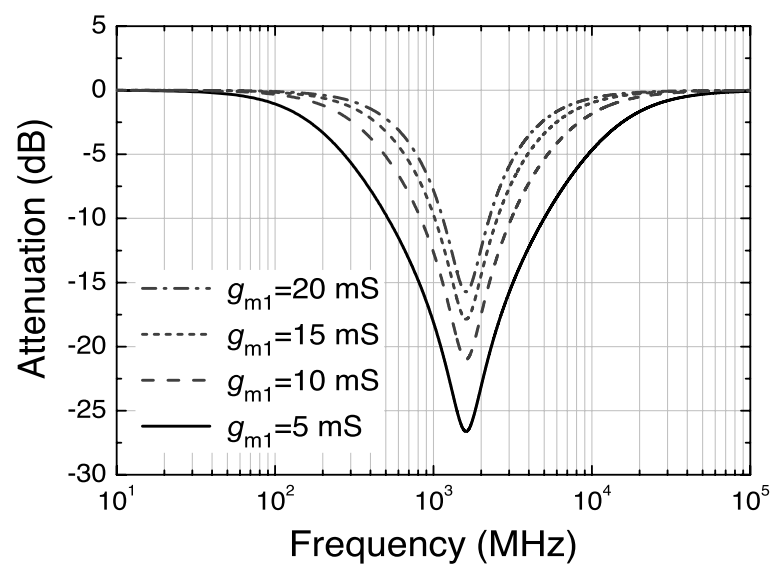

(b)

Fig. 2. (a) AC equivalent circuit of the RF tunable filter. (b) Calculated transfer characteristics of the front part of the filter, that is the source follower and the LC notch filter.

harmonic frequency. In addition, the remaining $9^{\text {th }}$ harmonic component is further suppressed by the LC notch filter. Compared to the previous cascaded Sallen-Key approach [5], it consumes less current and provides simple architecture.

Fig. 1 shows the circuit schematic. It consists of a source follower $\left(\mathrm{M}_{1,2}\right)$, an LC notch filter $\left(\mathrm{L}_{1}\right.$ and $\left.\mathrm{C}_{1}\right)$, and a Sallen-Key core $\left(\mathrm{M}_{3,4}, \mathrm{R}_{1,2}, \mathrm{C}_{2,3}\right)$. By switching $\mathrm{C}_{2}$ and $\mathrm{R}_{2}$, the corner frequency is tuned from 100 to $500 \mathrm{MHz}$ in five steps, and the transmission zero of the Sallen-Key stage is also controlled to be around $7^{\text {th }}$ harmonic frequency. In accordance with the corner frequency tuning, the LC notch frequency is also tuned to the $9^{\text {th }}$ harmonic frequency. In order to provide sufficient linearity with a large signal swing, the source follower is chosen as the filter's first stage due to its superior linearity. Even though the in-band gain of the source follower is slightly less than unity, it can be easily compensated by other blocks in the transmitter circuits via proper gain distribution.

The small signal equivalent circuit of the RF tunable filter is drawn in 
Fig. 2 (a). The transfer function of the source follower with the LC notch is given by

$$
\frac{v_{x}(s)}{v_{i}(s)}=\frac{s^{2}+\frac{R_{S}}{L_{1}} s+\frac{1}{L_{1} C_{1}}}{\alpha s^{2}+s \frac{1}{L_{1}}\left(\alpha R_{s}+g_{m 1}^{-1}\right)+\frac{\alpha}{L_{1} C_{1}}}
$$

where $\alpha=1+\left(g_{m 1} r_{o 1 S F}\right)^{-1}$ and, $r_{o 1 S F}=r_{o 1} \| r_{o S F}, \mathrm{R}_{\mathrm{S}}$ is the parasitic series resistance of $\mathrm{L}_{1}$, and $r_{o S F}$ is the output resistance of source follower's current source. And the transfer function of the subsequent Sallen-Key filter is given by

$$
\begin{aligned}
& \frac{v_{o}(s)}{v_{x}(s)} \\
& \quad=\frac{s^{2} C_{2} C_{3} R_{2} R_{o}+s C_{2} R_{o}+K}{s^{2} C_{2} C_{3}\left[R_{1} R_{2}+\left(R_{1}+R_{2}\right) R_{o}\right]+s\left[C_{3}\left(R_{1}+R_{2}\right)+C_{2}\left(R_{0}+R_{1}(1-K)\right)\right]+1}
\end{aligned}
$$

where $K=\frac{g_{m 3} r_{o 3 S K}}{1+g_{m 3} r_{o 3 S K}}, R_{o}=\frac{r_{o 3 S K}}{1+g_{m 3} r_{o 3 S K}}, r_{o 3 S K}=r_{o 3} \| r_{o S K}$, and $r_{o S K}$ is the output resistance of Sallen-Key stage's current source. It should be pointed out that the transconductance $g_{m 1}$ of the source follower $M_{1,2}$ must be carefully chosen to achieve optimum rejection characteristic by the LC notch. Eq. (1) indicates that the attenuation at the notch frequency $\omega_{0}=1 / \sqrt{\left(L_{1} C_{1}\right)}$ is $\mathrm{R}_{S} /\left(\alpha \mathrm{R}_{S}+1 / \mathrm{g}_{\mathrm{m}}\right)$ and the filter's $\mathrm{Q}$ factor is approximately given by $g_{m} \omega_{0} L_{1}$. Hence, lowering $g_{m}$ will increase the attenuation while degrading the filter's $Q$ and vice versa as illustrated in Fig. 2 (b). In this design, $\mathrm{g}_{\mathrm{m}}$ is optimally set to $16.8 \mathrm{mS}$ with $\mathrm{L}_{1}=2.6 \mathrm{nH}$ and $\mathrm{R}_{\mathrm{s}}=4.9 \Omega$, resulting in the peak attenuation of about $-17 \mathrm{~dB}$.

The RF tunable filter is fabricated in a $0.18-\mu \mathrm{m}$ CMOS process and integrated with a TV-band CMOS transmitter. Fig. 3 (a) shows the chip micrograph. The active area is $685 \times 450 \mu \mathrm{m}^{2}$. The top metal layer is used for the signal path for minimum parasitic capacitance with respect to the ground plane. And the signal line is drawn as short as possible in order to reduce path loss. The chip is packaged in a 48-pin leadless plastic chip carrier (LPCC) and tested on an evaluation board. The filter consumes $6.2 \mathrm{~mA}$ from a 1.8-V supply. Fig. 3 (b) shows the measured transfer characteristics. This measurement was performed with an added external amplifier to drive $50-\Omega$ load and its $12-\mathrm{dB}$ gain is de-embedded. The filter shows $2-\mathrm{dB}$ in-band loss. The cutoff frequency is tuned from 100 to $500 \mathrm{MHz}$ in five steps. The effect of LC notch filter on far-out stop-band attenuation is demonstrated in Fig. 3 (c) for the cases of 100- and 200-MHz cutoff frequencies. It can be observed that the far-out stop-band attenuation is significantly improved when the LC notch filter is activated. The maximum improvement of stop-band attenuation is almost $17 \mathrm{~dB}$ when the cutoff frequency is $100 \mathrm{MHz}$. As a result, in the final full transmitter circuit measurement [3], the $7^{\text {th }}$ harmonic component is found to be dramatically suppressed by $24.6 \mathrm{~dB}$ when the RF tunable filter is activated. Further measurements show that the in-band harmonics are always below $-42 \mathrm{dBc}$ across the full TV-band thanks to the significant 


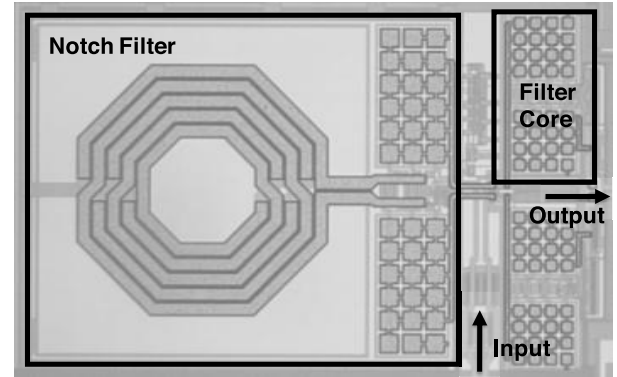

(a)

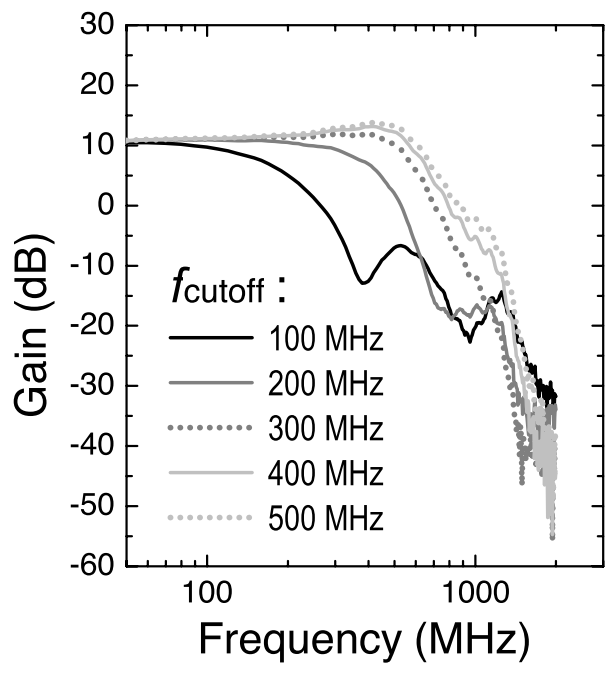

(b)

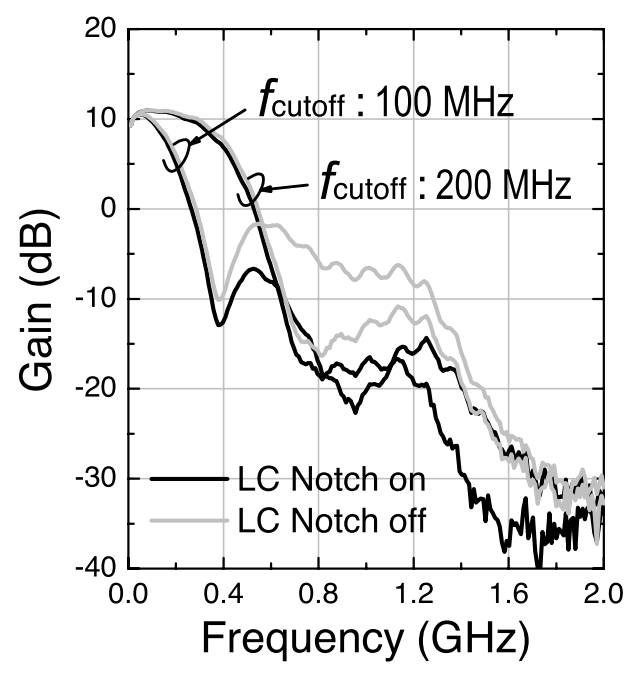

(c)

\begin{tabular}{l|l}
\hline Technology & $0.18 \mu \mathrm{m} \mathrm{CMOS}$ \\
Operating Frequency & $54 \sim 500 \mathrm{MHz}$ \\
Stop-band Attenuation & $>17 \mathrm{dBc}$ \\
In-band Harmonic Distortions & $<-42 \mathrm{dBc}$ (@ Tx output) \\
In-band Loss & $2 \mathrm{~dB}$ \\
Supply Voltage & $1.8 \mathrm{~V}$ \\
Current Consumption & $6.2 \mathrm{~mA}$ \\
\hline
\end{tabular}

(d)

Fig. 3. (a) Chip micrograph. (a) Measured filter transfer characteristics with cutoff frequency tuning. (b) Measured effects of the LC notch. (d) Performance summary of the filter.

contribution of the RF tunable filter. The measured performances of the RF tunable filter are summarized in Fig. $3(\mathrm{~d})$.

\section{Conclusion}

An RF tunable filter has been developed for TV white space transmitter applications. By combining the Sallen-Key structure and the LC notch filter, the $7^{\text {th }}$ and $9^{\text {th }}$ harmonic components are effectively suppressed with low current consumption. A small signal transfer function analysis is presented for finding the optimal design condition of the source follower followed by the LC notch. The measurement results prove that the proposed RF tunable filter can be successfully employed for the TV white space transmitter applications. 


\section{Acknowledgments}

This work has been supported by the Seoul Research and Business Development Program (GR070093) and "The Component Development of Ultra-Low Power ICTS" funded by the Ministry of Knowledge Economy. 